\title{
Elasto-static micropolar behavior of a chiral auxetic lattice
}

\author{
A. Spadoni ${ }^{\mathrm{a}, *}$, M. Ruzzene ${ }^{\mathrm{b}}$ \\ a Institute of Mechanical Engineering, École Polytechnique Fédérale de Lausanne (EPFL), Laboratory of Wave Mechanics and Multi-Field Interactions, \\ EPFL-STI-IGM-LOMI, ME B2 444, Station 9, 1015-CH Lausanne, Switzerland \\ b School of Aerospace Engineering, Georgia Institute of Technology, USA
}

\section{A R T I C L E I N F O}

\section{Article history:}

Received 2 May 2011

Received in revised form

24 August 2011

Accepted 7 September 2011

Available online 10 October 2011

\section{Keywords:}

Auxetic

Cellular solids

Chiral lattice

Negative Poisson's ratio

Couple-stress elasticity

\begin{abstract}
A B S T R A C T
Auxetic materials expand when stretched, and shrink when compressed. This is the result of a negative Poisson's ratio $v$. Isotropic configurations with $v \approx-1$ have been designed and are expected to provide increased shear stiffness $G$. This assumes that Young's modulus and $v$ can be engineered independently. In this article, a micropolarcontinuum model is employed to describe the behavior of a representative auxetic structural network, the chiral lattice, in an attempt to remove the indeterminacy in its constitutive law resulting from $v=-1$. While this indeterminacy is successfully removed, it is found that the shear modulus is an independent parameter and, for certain configurations, it is equal to that of the triangular lattice. This is remarkable as the chiral lattice is subject to bending deformation of its internal members, and thus is more compliant than the triangular lattice which is stretch dominated. The derived micropolar model also indicates that this unique lattice has the highest characteristic length scale $l_{c}$ of all known lattice topologies, as well as a negative first Lamé constant without violating bounds required for thermodynamic stability. We also find that hexagonal arrangements of deformable rings have a coupling number $N=1$. This is the first lattice reported in the literature for which couple-stress or Mindlin theory is necessary rather than being adopted a priori.
\end{abstract}

(c) 2011 Elsevier Ltd. All rights reserved.

\section{Introduction}

Auxetic materials have fascinated solid mechanicians ever since a polymeric foam with negative Poisson's ratio was introduced by Lakes (1987). Interest in these unique materials has since grown significantly and a number of auxetic configurations have been proposed, both chiral (Prall and Lakes, 1997; Grima et al., 2008) or not invariant with respect to reflections, and non-chiral (Gaspar et al., 2005; Lakes, 1991; Smith et al., 2000; Theocaris et al., 1997). Envisioned applications include indentation-resistant materials (Evans and Alderson, 2000; Lakes, 1987; Lakes and Elms, 1993; Smith et al., 1999), doubly curved panels with synclastic curvature (Evans, 1990; Evans and Alderson, 2000; Lakes, 1987), and miniaturized sandwich cores (Doyoyo and $\mathrm{Hu}, 2006$ ).

Materials with Poisson's ratio $v \approx-1$ are expected to display significant shear stiffness (Yang et al., 2004) and indentation resistance (Evans and Alderson, 2000; Lakes, 1987; Lakes and Elms, 1993; Smith et al., 1999). The employment of auxetic materials, at least isotropic ones, as indentation-resistant materials assumes that Young's modulus and Poisson's ratio can be designed independently (Evans and Alderson, 2000), while shear and bulk moduli are derived quantities. This approach ignores the fact that the microstructural design required to yield auxetic behavior may couple Young's modulus and Poisson's ratio. Poisson's ratio in fact is determined by anisotropy, non-affine deformations and internal rotational

\footnotetext{
* Corresponding author. Tel.: +41216933856.

E-mail address: alex.spadoni@epfl.ch (A. Spadoni).
} 
units (Lakes, 1991). The last two characteristics are achieved with cellular solids with a re-entrant microstructure (Choi and Lakes, 1995; Gibson and Ashby, 1997; Lakes, 1991; Lakes and Witt, 2002), one with concave cells, and chirality (Grima et al., 2008; Prall and Lakes, 1997).

Classical elasticity theory has been previously and successfully used to describe an equivalent continuum with auxetic properties (Gaspar et al., 2005; Grima et al., 2008; Lakes, 1991; Prall and Lakes, 1997; Smith et al., 2000; Theocaris et al., 1997). Negative Poisson's and increased shear resistance in fact do not describe the behavior of internal members but rather than of a bulk material made of may cells. These unique properties certainly encourage studies aimed at quantifying the benefits introduced by auxetic materials for different applications, but in may cases, it is found that $v=-1$. This leads to an indeterminate constitutive law.

In this work, a representative auxetic structural network with internal rotational units, known as the chiral lattice is investigated with an equivalent, micropolar-continuum model in an attempt to remove the indeterminacy $v=-1$ encountered so far (Prall and Lakes, 1997; Spadoni, 2008; Spadoni et al., 2009). A detailed microstructural analysis of the chiral lattice is also employed to describe the repercussions of auxetic behavior on the relationship between Young's modulus, shear modulus and Poisson's ratio. This lattice moreover features internal rotational units, in the form of rings, connected by tangent ligaments with rigid joints. This is important since the characteristics of ligament joints, rigid or deformable, determine the effective Poisson's ratio (Tanaka and Shibutani, 2009). In this work, rings with rigidly connected ligaments represent moment loci instead of force loci. Very different configurations ranging from a hexagonal packing of rings to the triangular lattice are obtained varying a single parameter known as the topology parameter which determines the ring radius. This provides the opportunity to define a micropolar model where (i) the contribution of rotational units can be tailored, as well as (ii) a model that can connect bending and axial-dominated microstructural configurations. These two deformation mechanisms indeed distinguish auxetic and classic media $(v \geq 0)$ with rigid joints between members.

Following a description of geometric properties in Section 2, the micropolar constitutive law for a general isotropic medium is presented in Section 3 and its application for this particular framework is discussed. Two configurations are employed to study the auxetic behavior of the chiral lattice in Section 4. In the first case, rings are assumed rigid; this simplification enables the derivation of micropolar elastic constants analytically. A second case with deformable rings, the actual behavior of the lattice, is analyzed with a finite-element model. Finally, the mechanical properties of both models are compared to those of other common cellular solids like the square, triangular a hexagonal configurations.

\section{Hexagonal chiral lattice}

The structural layout of a hexagonal chiral lattice shown in Fig. 1 consists of rings of radius $r$, acting as nodes, connected by ribs or ligaments, of length $L$ tangent to the nodes themselves. The distance between node centers is denoted as $R$, while the angle between the imaginary line connecting the node centers and the ribs is defined as $\beta$. The angle between adjacent ligaments is denoted as $2 \theta$. The wall thickness of nodes and ribs is denoted as $t_{c}$ and $t_{b}$ respectively. As described in Prall and Lakes (1997), the following geometric relationships hold:

$$
\sin \beta=\frac{2 r}{R}, \quad \tan \beta=\frac{2 r}{L}, \quad \sin \theta=\frac{R / 2}{R}, \quad \cos \beta=\frac{L}{R} .
$$

The tessellation of the unit cell of Fig. 1 a along the symmetry vectors $\boldsymbol{e}_{1}$ and $\boldsymbol{e}_{2}$ generates the lattice shown in Fig. $1 \mathrm{~b}$. The symmetry vectors shown in Fig. $1 \mathrm{~b}$ are one of the three possible sets, given hexagonal symmetry. A convenient set is

$$
\begin{aligned}
& \boldsymbol{e}_{1}=\{R \cos \theta, R \sin \theta\}^{T}, \\
& \boldsymbol{e}_{2}=\{-R \cos \theta, R \sin \theta\}^{T} .
\end{aligned}
$$

a

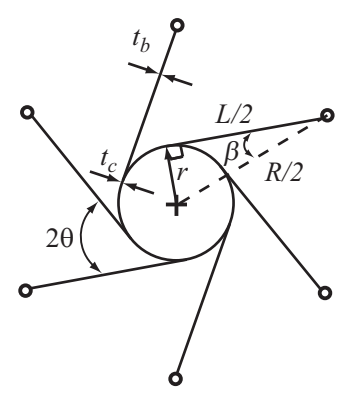

b

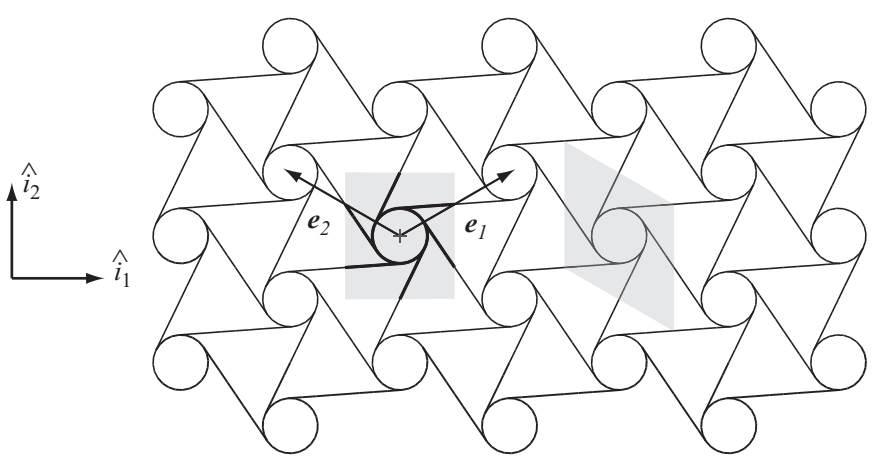

Fig. 1. Geometry of a hexagonal chiral lattice: (a) unit cell and (b) unit volume with symmetry vectors. 
a

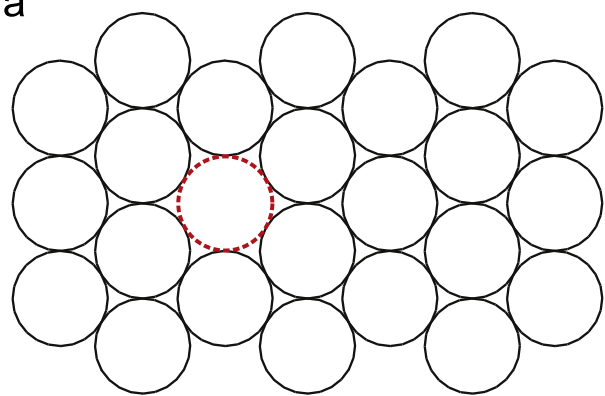

C

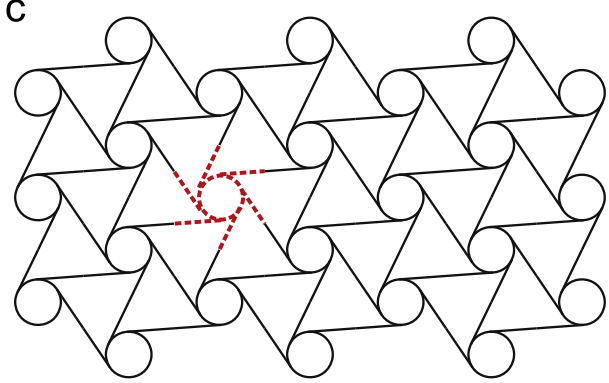

b

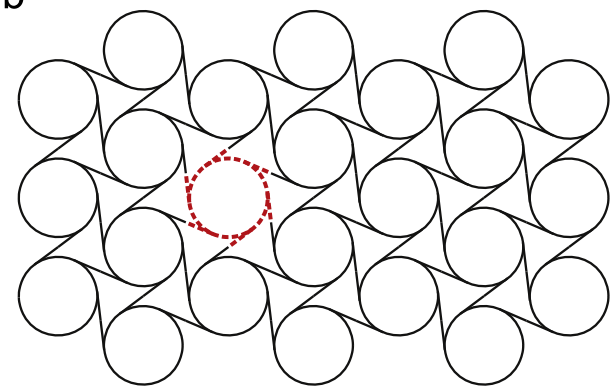

d

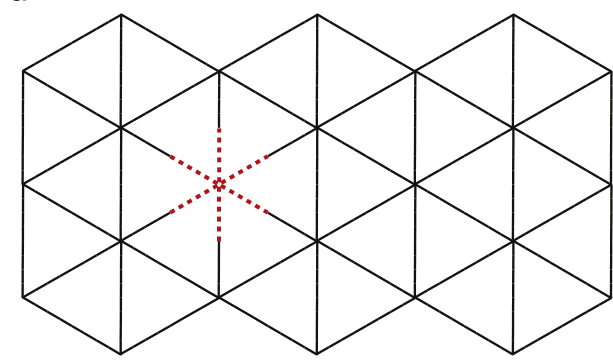

Fig. 2. Lattice configurations corresponding to increasing topology parameter $L / R$. (a) $L / R \rightarrow 0$. (b) $L / R=0.60$. (c) $L / R=0.90$. (d) $L / R \rightarrow 1$. Dashed lines denote the unit cell for each configuration.

For the remainder of this manuscript, vectors are denoted as bold, lower case letters, while capital bold letters identify matrices.

Significantly different configurations can be obtained by varying the ratio $L / R$ here denoted as the topology parameter. Possible topologies range from a hexagonal arrangement of rings $(L / R \rightarrow 0)$ to the classic triangular lattice $(L / R \rightarrow 1)$ with associated unit cells highlighted in dashed lines in Fig. 2. The ability to generate such topologically and mechanically different structural networks provides the unique opportunity of deriving a mechanical model that connects bendingdominated, axially-dominated, conventional, and auxetic lattices as a continuous function of the topology parameter. The same model could also bring a very interesting insight into the transition between bending-dominated behavior and axially dominated behavior which depend on ligament-wall thickness very differently. This implies that the relative density plays an important role in determining the mechanical response of the chiral assembly. The relative density $\bar{\rho}$ of a cellular solid is defined as the volume occupied by solid phase, in this case the walls of a unit cell, normalized by the sum of the volumes of all phases. The second phase is simply vacuum in this case, with no influence on the static or and dynamic response of the structure. Assuming that the lattice's walls are thin, the relative density is

$$
\bar{\rho}=\frac{\rho^{*}}{\rho_{s}}=\frac{2 \pi r t_{c}+6 L t_{b} / 2}{R^{2} \cos \theta},
$$

where $\rho^{*}$ is the equivalent density of the lattice, while $\rho_{\mathrm{s}}$ is the density of the constituent material. Furthermore, since from Eq. (1) $\theta$ is always $30^{\circ}$, the relative density may be recast as

$$
\bar{\rho}=\frac{\rho^{*}}{\rho_{s}}=\frac{2 \sqrt{3}\left(2 \pi r t_{c}+3 L t_{b}\right)}{3 R^{2}} .
$$

\section{Constitutive model}

A treatment of a micropolar constitutive model for noncentrosymmetric media, or configurations that are not invariant to reflections, has eloquently been presented by Lakes and Benedict (1982). They found that in a noncentrosymmetric medium described by a micropolar elasticity model, direct and coupled stresses are connected by three elastic constants. If plane deformations only are considered, as is the case here, direct and couple stresses are independent of each other. This can be shown by considering the strain energy density $\Psi$ for a general micropolar continuum with no residual or latent stress as (Eringen, 2001; Lakes and Benedict, 1982)

$$
\Psi=\frac{1}{2} A_{i j k l} \varepsilon_{k l} \varepsilon_{i j}+\frac{1}{2} B_{i j k l} \varepsilon_{i j} k_{k l}+C_{i j k l} k_{i j} k_{k l},
$$

where $\varepsilon_{i j} \equiv u_{j, i}-e_{k i j} \phi_{k}$ is the micropolar strain tensor, $u_{i}$ is the displacement vector, $\phi_{k}$ is the microrotation vector, $e_{k i j}$ is the Levi-Civita permutation symbol, and $k_{k l} \equiv \phi_{l, k}$ is the curvature tensor. The subscript ( $)_{i}$ indicates a partial derivative with 
respect to coordinate $i$ while $A_{i j k l}, B_{i j k l}$ and $C_{i j k l}$ are fourth-order tensors of elastic constants. Symmetries in the employed model result in $A_{i j k l}=A_{k l i j}$ and $C_{i j k l}=C_{k l i j}$ (Eringen, 2001). The usual Einstein summation convention is used. The resulting constitutive model becomes (Eringen, 2001)

$$
\begin{gathered}
\sigma_{i j}=\frac{\partial \Psi}{\partial \varepsilon_{i j}}=A_{i j k l} \varepsilon_{k l}+B_{i j k l} k_{k l}, \\
m_{i j}=\frac{\partial \Psi}{\partial k_{i j}}=B_{k l i j} \varepsilon_{k l}+C_{i j k l} k_{k l},
\end{gathered}
$$

where $\sigma_{k l}$ and $m_{k l}$ are the direct and couple stress tensors respectively. Lakes and Benedict (1982) conclude that reflections invert the sign of the microrotation vector $\phi_{k}$, and thus the strain energy density $\Psi$ is not invariant to such operations. This is certainly the case of the noncentrosymmetric lattice in question, appropriately termed chiral. As a result $B_{i j k l} \neq 0$ and $C_{i j k l} \neq 0$.

Given the hexagonal symmetry of the chiral lattice, its mechanical behavior is expected to be isotropic (Love, 1927, Chapter VI, Section 110). The constitutive tensors $A_{i j k l}, B_{i j k l}$ and $C_{i j k l}$ can be simplified considering the most general form of a fourth-order tensor (one with proper isotropy but not necessarily mirror symmetry):

$$
T_{i j k l}=T_{1} \delta_{i j} \delta_{k l}+T_{2} \delta_{i k} \delta_{j l}+T_{3} \delta_{i l} \delta_{j k}
$$

where $\delta_{i j}$ is the kronecker delta. The stress tensors become

$$
\begin{aligned}
& \sigma_{i j}=A_{1} \varepsilon_{r r} \delta_{i j}+A_{2} \varepsilon_{i j}+A_{3} \varepsilon_{j i}+B_{1} k_{r r} \delta_{i j}+B_{2} k_{i j}+B_{3} k_{j i}, \\
& m_{i j}=B_{1} \varepsilon_{r r} \delta_{i j}+B_{2} \varepsilon_{i j}+B_{3} \varepsilon_{j i}+C_{1} k_{r r} \delta_{i j}+C_{2} k_{i j}+C_{3} k_{j i} .
\end{aligned}
$$

For the lattice under consideration, 2D plane deformations are of interest. Accordingly $\varepsilon=\left\{\varepsilon_{11} \varepsilon_{22} \varepsilon_{12} \varepsilon_{21}\right\}^{T}, \phi=\phi_{3}$, $\boldsymbol{\sigma}=\left\{\begin{array}{llll}\sigma_{11} & \sigma_{22} & \sigma_{12} & \sigma_{21}\end{array}\right\}^{T}$ and $\boldsymbol{m}=\left\{m_{13} m_{23}\right\}$. In this case, $B_{i j k l}=C_{1}=0$ and Eqs. (9) and (10) collapse to those of an isotropic micropolar medium with mirror symmetry. Within this framework, the noncentrosymmetric character of the chiral lattice simply produces moments about nodes or rings, and in turn the couple stress components $m_{13}$ and $m_{31}$. The auxetic behavior of the structural network, however, is not lost and remains the focus of this work.

\section{Elasto-static behavior}

The dominant deformation mechanism in 2D structural lattices may be of extensional or bending nature, according to the manner in which the lattice's components are interconnected. A rigorous procedure based on Maxwell's theorem to determine the deformation mechanism that defines a given 2D lattice is presented by Deshpande et al. (2001). This is a defining characteristic since equivalent stiffness for extensional lattices scales as $\bar{\rho}$, where $\bar{\rho}$ is the relative density, as opposed to $\bar{\rho}^{3}$ for bending-dominated lattices. For a given relative density $\bar{\rho}$, axially-dominated configurations are much stiffer.

The triangular lattice for example is characterized by axial deformations of its internal components (Wang and McDowell, 2004), while the most common lattice, the hexagonal lattice, is dominated by cell-wall bending (Gibson and Ashby, 1997). The same bending-dominated behavior has been observed experimentally for the chiral lattice (Prall and Lakes, 1997) (numerically reproduced in Fig. 3). Knowledge of the equivalent mechanical properties of the chiral lattice, however, is currently very limited; the only investigations available in the literature are those by Prall and Lakes (1997) and Spadoni (2008). The first analysis by Prall and Lakes (1997) investigated both Young's modulus and Poisson's ratio in the plane with the following assumptions:

1. nodes (or rings) are considered rigid;

2. internal forces oriented in a direction perpendicular to the externally applied stress vanish;

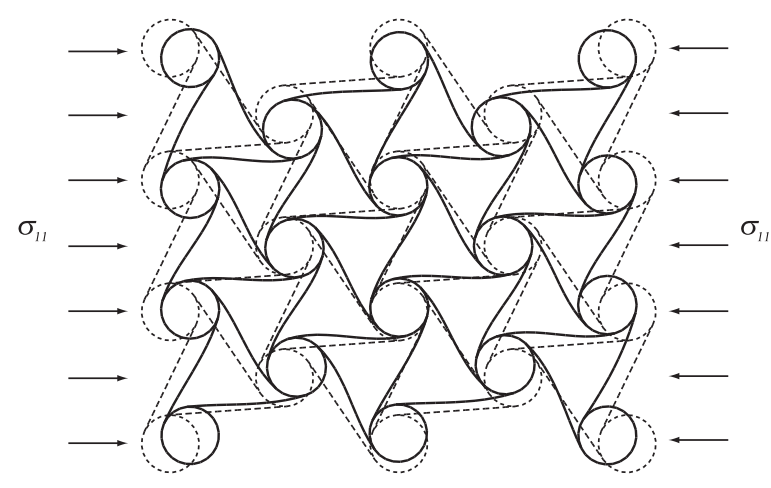

Fig. 3. Deformed configuration resulting from uniaxial stress. 
3. internal forces are dictated by the experimentally observed kinematic behavior;

4. axial and shear deformations of the ligaments are neglected;

5. deformations are small.

In their theoretical account, Prall and Lakes (1997) find Poisson's ratio $v=-1$. This implies that the in-plane shear modulus is infinite and the resulting constitutive tensors of Eqs. (9) and (10) are indefinite. In an attempt to employ a homogenized model of the chiral lattice to explore possible applications such as chiral honeycombs, Spadoni (2008) revisited the analysis by relaxing some of the above assumptions within the Cauchy elasticity model.

In order to define a more accurate constitutive model and study variations in mechanical behavior varying with the size of rings (Fig. 2), the following analyses are here extended to a generalized micropolar continuum and, as in Spadoni (2008), the above assumptions are relaxed by studying two configurations: in the first case (case 1), assumptions 2-4 are removed, while in a second case (case 2 ) the only retained assumption is 5 .

\subsection{Case 1: rigid nodes}

In the original study by Prall and Lakes (1997), a single ligament was analyzed in light of isotropy and experimental observations indicating that all ligaments deformed equally. The dominant behavior was observed to be a sigmoidal deformation of the ligaments winding onto the rings, resulting in the rings themselves rolling along $R$ (Prall and Lakes, 1997). The single-ligament model was also employed in Spadoni (2008) leading to a Poisson's ratio $v=-1$. In the current framework, however, a refined estimate of the same parameter is desired even for rigid rings. Given the rotational symmetry of the unit cell depicted in Fig. 1a, the irreducible set of distinct mechanical entities of Fig. 4a is expected to produce the elasto-static behavior of the entire lattice. Constraint equations relating the kinematics of the center of each ring to those of material points on the tings themselves (Fig. 4a) are expressed as

$$
\begin{aligned}
& \boldsymbol{x}_{\mathrm{OA}}=\boldsymbol{x}_{\mathrm{OB}}+\boldsymbol{x}_{\mathrm{BA}}=\boldsymbol{x}_{\mathrm{OB}}+r\left(\cos \psi \hat{\boldsymbol{i}}_{1}+\sin \psi \hat{\boldsymbol{i}}_{2}\right), \\
& \boldsymbol{x}_{\mathrm{OA}}=\boldsymbol{x}_{\mathrm{OB}}+\left(u_{\mathrm{B}} \hat{\boldsymbol{i}}_{1}+v_{\mathrm{B}} \hat{\boldsymbol{i}}_{2}\right)+\boldsymbol{x}_{\mathrm{BA}}=\boldsymbol{x}_{\mathrm{OB}}+\left(u_{\mathrm{B}} \hat{\boldsymbol{i}}_{1}+v_{\mathrm{B}} \hat{\boldsymbol{i}}_{2}\right)+r\left[\cos (\psi+\phi) \hat{\boldsymbol{i}}_{1}+\sin (\psi+\phi) \hat{\boldsymbol{i}}_{2}\right], \\
& \left(u_{\mathrm{A}} \hat{\boldsymbol{i}}_{1}+v_{\mathrm{A}} \hat{\boldsymbol{i}}_{2}\right)=\boldsymbol{x}_{\mathrm{OA}}-\boldsymbol{x}_{\mathrm{OA}}=\left(u_{\mathrm{B}} \hat{\boldsymbol{i}}_{1}+v_{\mathrm{B}} \hat{\boldsymbol{i}}_{2}\right)+r[\cos (\psi+\phi)-\cos \psi] \hat{\boldsymbol{i}}_{1}+r[\sin (\psi+\phi)-\sin \psi] \hat{\boldsymbol{i}}_{2} \\
& \approx\left(u_{\mathrm{B}} \hat{\boldsymbol{i}}_{1}+v_{\mathrm{B}} \hat{\boldsymbol{i}}_{2}\right)-r \phi \sin \psi \hat{\boldsymbol{i}}_{1}+r \phi \cos \psi \hat{\boldsymbol{i}}_{2} .
\end{aligned}
$$

Referring to Fig. $4 \mathrm{~b},\left(u_{\mathrm{A}}, v_{\mathrm{A}}\right)$ are the displacement components of point $\mathrm{A}$ along $\hat{\boldsymbol{i}}_{1}$ and $\hat{\boldsymbol{i}}_{2}$ respectively, while $\left(u_{\mathrm{B}}, v_{\mathrm{B}}\right)$ are those of point B, $\phi$ is the rotation of the ring, and $\boldsymbol{x}$ denotes a position vector. The resulting kinematic constraint of Eq. (13) is linearized in compliance with the assumption of small displacements and rotations. The same constraint may be expressed in matrix form as:

$$
\left\{\begin{array}{l}
u_{\mathrm{A}} \\
v_{\mathrm{A}} \\
\phi_{\mathrm{A}}
\end{array}\right\}=\left[\begin{array}{ccc}
1 & 0 & -r \sin \psi \\
0 & 1 & r \cos \psi \\
0 & 0 & 1
\end{array}\right]\left\{\begin{array}{c}
u_{\mathrm{B}} \\
v_{\mathrm{B}} \\
\phi_{\mathrm{B}}
\end{array}\right\}=\boldsymbol{T}(\psi)\left\{\begin{array}{l}
u_{\mathrm{B}} \\
v_{\mathrm{B}} \\
\phi_{\mathrm{B}}
\end{array}\right\} .
$$

a

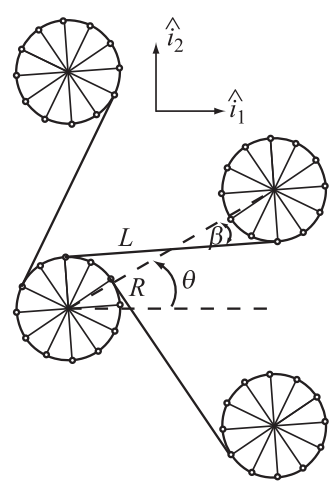

b

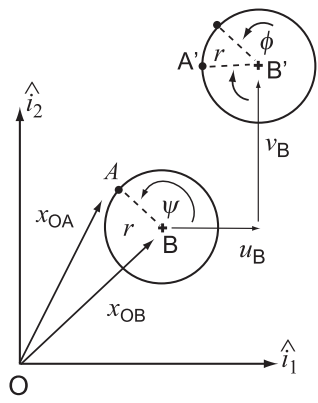

C

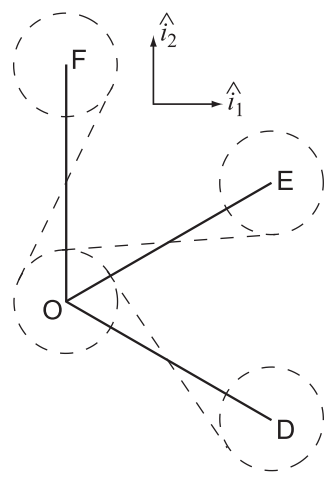

Fig. 4. Irreducible set of entities required to study the mechanical response of the chiral lattice: geometry of ligaments connecting rigid rings (a), kinematics of a rigid ring (b), and equivalent mechanical model (c). 
The relation between forces and displacements for a beam-like entity such as a ligament including axial and bending deformations is

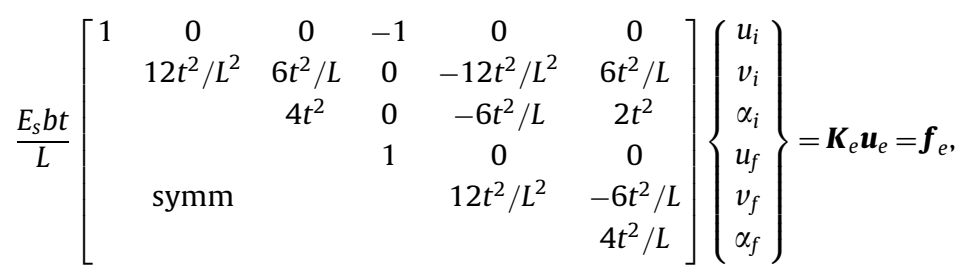

where $E_{s}$ is Young's modulus of the constituent material while $\boldsymbol{K}_{e}, \boldsymbol{u}_{e}$ and $\boldsymbol{f}_{e}$ are the symmetric stiffness matrix, vector of generalized displacements and end forces. The subscript ( $)_{e}$ indicates quantities expressed in the element (local) coordinate system. Upon projection of generalized displacements to the global coordinate system and assembly of the global stiffness matrix $\boldsymbol{K}$ containing the contributions all the three ligaments, the simplified geometric model shown in Fig. 4a can be reduced to that depicted in Fig. 4c by way of Eq. (14) as follows:

$$
\tilde{\boldsymbol{K}} \tilde{\boldsymbol{u}}=\boldsymbol{T}(\psi)^{T} \boldsymbol{K} \boldsymbol{T}(\psi) \tilde{\boldsymbol{u}}=\boldsymbol{T}(\psi)^{T} \boldsymbol{f},
$$

where ( ) indicates quantities associated with the reduced model depicted in Fig. 4c. The strain energy density of a unit cell can be computed as

$$
\Psi=\frac{2}{2 V} \cdot \frac{1}{2} \tilde{\boldsymbol{u}}^{T} \tilde{\boldsymbol{K}} \tilde{\boldsymbol{u}},
$$

where the factor 2 is needed as the frame assembly of Fig. 4a represents half of the complete unit cell. The volume of one unit cell is $V=b R^{2} \cos \theta$ comprising ligaments of length $L / 2$. The modeled system of Fig. 4, however, has ligaments of length $L$. The strain energy in Eq. (17) is thus associated with $2 V$. The elastic constants $A_{k l m n}$ and $C_{k l m n}$ are evaluated as

$$
A_{i j k l}=\frac{\partial^{2} \Psi}{\partial \varepsilon_{k l} \partial \varepsilon_{i j}}, \quad C_{i j k l}=\frac{\partial^{2} \Psi}{\partial k_{k l} \partial k_{i j}} .
$$

In order to obtain the strain energy of a unit cell in terms of geometric and material properties, the following displacement field is assumed:

$$
\begin{aligned}
& u_{\mathrm{O} i}(x, y)=u\left(x_{\mathrm{O}}, y_{\mathrm{O}}\right)+\Delta x \varepsilon_{11}+\Delta y\left(\varepsilon_{21}-\phi\right)+O\left(\Delta x^{2}, \Delta y^{2}\right), \\
& v_{\mathrm{O} i}(x, y)=v\left(x_{0}, y_{\mathrm{O}}\right)+\Delta y \varepsilon_{22}+\Delta x\left(\varepsilon_{12}+\phi\right)+O\left(\Delta x^{2}, \Delta y^{2}\right), \\
& \phi_{\mathrm{O} i}(x, y)=\phi\left(x_{0}, y_{\mathrm{O}}\right)+\Delta x \phi_{, 1}+\Delta y \phi_{, 2}+O\left(\Delta x^{2}, \Delta y^{2}\right),
\end{aligned}
$$

where, from Eq. (2), $|\Delta x|=R \cos \theta$ and $|\Delta y|=R \sin \theta$. Upon enforcing the rigid-node constraint of Eq. (16), the degree of freedom $\alpha$ in Eq. (15) is the finite rotation of a rigid ring, and it is associated with global deformations of the ligaments (winding behavior in Fig. 3), different from the micro-rotation $\phi$. For the assumed strain field of Eqs. (19)-(21), $\alpha$ is thus not known a priori and must be solved for. Since the sub-assembly of Fig. 4a is part of an infinite lattice, all rings must rotate by the same amount for a uniform strain field. This constraint can be enforced by relating the set of constrained displacements $\hat{\boldsymbol{u}}$ to $\tilde{\boldsymbol{u}}$ with a boolean matrix $\boldsymbol{T}_{B}$ such that $\hat{\boldsymbol{u}}=\boldsymbol{T}_{B} \tilde{\boldsymbol{u}}$. The associated stiffness matrix is obtained as $\hat{\boldsymbol{K}}=\boldsymbol{T}_{B}^{T} \tilde{\boldsymbol{K}} \boldsymbol{T}_{B}$. Dividing Eq. (16) into known $k$ and unknown $u$ entities as

$$
\left[\begin{array}{ll}
\hat{\boldsymbol{K}}_{k k} & \hat{\boldsymbol{K}}_{k u} \\
\hat{\boldsymbol{K}}_{u k} & \hat{\boldsymbol{K}}_{u u}
\end{array}\right]\left\{\begin{array}{l}
\hat{\boldsymbol{u}}_{k} \\
\hat{\boldsymbol{u}}_{u}
\end{array}\right\}=\left\{\begin{array}{l}
\hat{\boldsymbol{f}}_{u} \\
\hat{\boldsymbol{f}}_{k}
\end{array}\right\} .
$$

The rotations of the rigid rings can be evaluated as

$$
\hat{\boldsymbol{u}}_{u}=\hat{\boldsymbol{K}}_{u u}^{-1}\left(\hat{\boldsymbol{K}}_{u k} \hat{\boldsymbol{u}}_{k}\right)
$$

given the assumed displacement field of Eqs. (19)-(21) expressed as $\tilde{\boldsymbol{u}}_{k}$. The constitutive laws of Eqs. (9) and (10) can be re-arranged in matrix form as

$$
\left\{\begin{array}{l}
\sigma_{11} \\
\sigma_{22} \\
\sigma_{12} \\
\sigma_{21} \\
m_{13} \\
m_{23}
\end{array}\right\}=\left[\begin{array}{cccccc}
D_{11} & D_{12} & 0 & 0 & 0 & 0 \\
D_{21} & D_{22} & 0 & 0 & 0 & 0 \\
0 & 0 & D_{33} & D_{34} & 0 & 0 \\
0 & 0 & D_{43} & D_{44} & 0 & 0 \\
0 & 0 & 0 & 0 & D_{55} & 0 \\
0 & 0 & 0 & 0 & 0 & D_{66}
\end{array}\right]\left\{\begin{array}{l}
\varepsilon_{11} \\
\varepsilon_{22} \\
\varepsilon_{12} \\
\varepsilon_{21} \\
\kappa_{13} \\
\kappa_{23}
\end{array}\right\}
$$


These elastic constants for the chiral lattice are

$$
\begin{aligned}
& D_{11}=\frac{\sqrt{3} E_{s} t}{4 L^{3}} \frac{\left(t^{4}-L^{4}\right) \cos ^{2} \beta+L^{4}+3 L^{2} t^{2}}{\left(t^{2}-L^{2}\right) \cos ^{2} \beta+L^{2}}, \\
& D_{12}=-\frac{\sqrt{3} E_{s} t}{4 L^{3}} \frac{L^{4} \tan ^{2} \beta+t^{4}-L^{2} t^{2}\left(1+\tan ^{2} \beta\right)}{L^{2} \tan ^{2} \beta+t^{2}}, \\
& D_{21}=-\frac{\sqrt{3} E_{s} t}{4 L^{3}} \frac{L^{4} \tan ^{2} \beta+t^{4}-L^{2} t^{2}\left(1+\tan ^{2} \beta\right)}{L^{2} \tan ^{2} \beta+t^{2}}, \\
& D_{22}=\frac{\sqrt{3} E_{s} t}{4 L^{3}} \frac{\left(t^{4}-L^{4}\right) \cos ^{2} \beta+L^{4}+3 L^{2} t^{2}}{\left(t^{2}-L^{2}\right) \cos ^{2} \beta+L^{2}}, \\
& D_{33}=\frac{\sqrt{3} E_{s} t}{4 L^{3}} \frac{\left[L^{4} 2 \tan ^{2} \beta+L^{2} R^{2}+t^{2}\left(2 L^{2}+R^{2}\right)\right]}{R^{2}}, \\
& D_{34}=-\frac{\sqrt{3} E_{s} t}{4 L^{3}} \frac{\left[L^{4} 2 \tan ^{2} \beta-L^{2} R^{2}+t^{2}\left(2 L^{2}-R^{2}\right)\right]}{R^{2}}, \\
& D_{43}=-\frac{\sqrt{3} E_{s} t}{4 L^{3}} \frac{\left[L^{4} 2 \tan ^{2} \beta-L^{2} R^{2}+t^{2}\left(2 L^{2}-R^{2}\right)\right]}{R^{2}}, \\
& D_{44}=\frac{\sqrt{3} E_{s} t}{4 L^{3}} \frac{\left[L^{4} 2 \tan ^{2} \beta+L^{2} R^{2}+t^{2}\left(2 L^{2}+R^{2}\right)\right]}{R^{2}}, \\
& D_{55}=\frac{\sqrt{3} E_{s} t}{12 L}\left(3 L^{2} \tan ^{2} \beta+4 t^{2}\right), \\
& D_{66}=\frac{\sqrt{3} E_{s} t}{12 L}\left(3 L^{2} \tan ^{2} \beta+4 t^{2}\right) .
\end{aligned}
$$

Note that all elastic constants relating direct stresses to direct strains have the leading-order term $(t / L)^{3}$, which, in light of Eq. (4), confirms that the behavior of the chiral lattice is bending dominated. The contribution of microrotations to couple stresses, however, is dominated by axial deformations of the ligaments as $D_{55}=D_{66} \propto(t / L)$ indicating a very strong micropolar behavior. The chiral lattice, as for the triangular lattice, is isotropic in plane. This was concluded on the basis of the hexagonal symmetry in Section 3 and it is here confirmed by the derived elastic constants. The hexagonal arrangement of rings (Fig. 2a) cannot be analyzed within the current assumptions as all strain energy would be stored in the rings themselves. The mechanical behavior obtained assuming rigid rings is applicable for $L / R \in(0,1]$ and it will be in significant error as $L / R \rightarrow 0$.

For the topology parameter $L / R=1(\beta=0)$, one obtains the equilateral triangular lattice (Fig. $2 \mathrm{~d}$ ), and the elastic constants of Eqs. (25)-(34) become

$$
\begin{aligned}
& D_{11 T}=\frac{\sqrt{3} E_{s} t}{4 L^{3}}\left(3 L^{2}+t^{2}\right), \\
& D_{12 T}=\frac{\sqrt{3} E_{s} t}{4 L^{3}}\left(L^{2}-t^{2}\right), \\
& D_{21 T}=\frac{\sqrt{3} E_{s} t}{4 L^{3}}\left(L^{2}-t^{2}\right), \\
& D_{22 T}=\frac{\sqrt{3} E_{s} t}{4 L^{3}}\left(3 L^{2}+t^{2}\right), \\
& D_{33 T}=\frac{\sqrt{3} E_{s} t}{4 L^{3}}\left(L^{2}+3 t^{2}\right), \\
& D_{34 T}=\frac{\sqrt{3} E_{s} t}{4 L^{3}}\left(L^{2}-t^{2}\right), \\
& D_{43 T}=\frac{\sqrt{3} E_{s} t}{4 L^{3}}\left(L^{2}-t^{2}\right),
\end{aligned}
$$




$$
\begin{aligned}
& D_{44 T}=\frac{\sqrt{3} E_{s} t}{4 L^{3}}\left(L^{2}+3 t^{2}\right), \\
& D_{55 T}=\frac{\sqrt{3} E_{s} t^{3}}{3 L}, \\
& D_{66 T}=\frac{\sqrt{3} E_{s} t^{3}}{3 L},
\end{aligned}
$$

which are identical to those obtained for a triangular lattice in Kumar and McDowell (2004) and derived without considering second or higher-order terms in the Taylor-series expansion of displacements of Eqs. (19)-(21). This is an encouraging result as it confirms the continuity in mechanical behavior for evolving configurations with respect to $L / R$ (Fig. 2), and it provides the opportunity to contrast the elastic behavior of bending and axially dominated configurations.

A more intuitive prospective of the chiral lattice behavior is obtained by defining engineering constants. Following the treatments in Nakamura and Lakes (1995) and Yang and Huang (2001), the micropolar Young's modulus and micropolar Poisson's ratio are defined as $E_{m}=\left(D_{11}^{2}-D_{12}^{2}\right) / D_{11}$ and $v_{m}=D_{12} / D_{11}$ and are obtained assuming the same relationships as for plane deformations in classical elasticity:

$$
\begin{aligned}
& \bar{E}_{m}=\frac{E_{m}}{E_{s}}=\frac{4 \sqrt{3}\left[1+(t / L)^{2}\right]}{\left[2(t / L)^{4} \cos ^{2} \beta+2 \sin ^{2} \beta+6(t / L)^{2}\right]}\left(\frac{t}{L}\right)^{3}, \\
& v_{m}=\frac{4(t / L)^{2}}{(t / L)^{4} \cos ^{2} \beta+1-\cos ^{2} \beta+3(t / L)^{2}}-1 .
\end{aligned}
$$

The stiffness modulus scales with $(t / L)^{3}$ confirming the bending-dominated behavior of the chiral lattice. In addition, Young's modulus smoothly changes to that of the axially dominated triangular lattice (Wang and McDowell, 2004) (where it is derived disregarding bending, thus no $(t / L)^{2}$ terms)

$$
\frac{E_{m, T}}{E_{s}}=\frac{2 \sqrt{3}\left[1+(t / L)^{2}\right]}{\left[3+(t / L)^{2}\right]}\left(\frac{t}{L}\right)
$$

and $v_{m, T}=1 / 3$. Poisson's ratio (Eq. (46)) is close to -1 but increases with increasing ligament aspect ratio $t / L$, hence the indeterminacy in elastic constants is removed. Poisson's ratio in fact would be exactly -1 only if $t / L=0$. It also varies smoothly as $L / R \rightarrow 1(\beta \rightarrow 0)$.

While the micropolar shear modulus $G_{m}$ can simply be obtained from Young's modulus and Poisson's ratio above, it is worth presenting it explicitly as auxetic or negative Poisson's-ratio materials are often touted for their expected large shear stiffness (Alderson et al., 1994; Evans and Alderson, 2000; Lakes and Elms, 1993). From Eqs. (45) and (46), the shear stiffness is

$$
\bar{G}_{m}=\frac{G_{m}}{E_{s}}=\frac{\sqrt{3}}{4}\left(\frac{t}{L}\right)+\frac{\sqrt{3}}{4}\left(\frac{t}{L}\right)^{3} .
$$

The shear modulus does not depend on the topology of the chiral lattice, i.e. it does not depend on the angle $\beta$ in turn determined by $L / R$, and it is the same as that of the triangular lattice reported in Wang and McDowell (2004) except for the higher order term $(t / L)^{3}$. Moreover, it scales with the ligament's aspect ratio $(t / L)$ indicating that shear behavior is determined by axial deformations of the ligaments. This leads to the conclusion that, while a 2D medium with $v \approx-1$ indeed presents high shear stiffness, the deformation mechanism necessary to achieve strong isotropic, auxetic behavior limits the shear stiffness to that of a medium with axially dominated deformations. This in turn restricts auxetic materials, at least in 2D, to be very efficient systems for shear stiffness relatively to bending-dominated media and not in an absolute sense.

The chiral lattice, however, remains a unique structural arrangement to study the strength of micropolar behavior as rings decrease in size, or $L / R \rightarrow 1$. This can be investigated with two more micropolar constants: namely the characteristic length $l_{c}^{2}=D_{55} /\left(4 G_{m}\right)$ and the coupling number $N^{2}=\left(D_{33}-D_{34}\right) /\left(2 D_{33}\right)$ (Nakamura and Lakes, 1995; Yang and Huang, 2001)

$$
\begin{aligned}
& l_{c}^{2}=R^{2} \cos ^{2} \beta \frac{\left[\frac{1}{4} \tan \beta+\frac{1}{3}(t / L)^{2}\right]}{\left[1+(t / L)^{2}\right]}, \\
& N^{2}=1-\frac{\left[1+(t / L)^{2}\right]}{\left[3+(t / L)^{2}-2 \cos ^{2} \beta+2(t / L)^{2} \cos ^{2} \beta\right]} .
\end{aligned}
$$

The micropolar characteristic length $l_{c}$ directly scales with the lattice parameter $R$ and it is weakly dependent on ligament aspect ratio $t / L$ or in turn on relative density. For elasto-static behavior, the characteristic length affects the decay rate of internal stresses from tractions at the boundaries of a body (Chen and Fleck, 2002; Diebels and Steeb, 2002; Eringen, 2001; Nakamura and Lakes, 1995) and also determines the magnitude of couple stresses (see Nakamura and Lakes, 1995, 
Eq. (14)). The coupling number $N$ determines the strength of micropolar behavior. Both $l_{c}$ and $N$ are discussed in more detail in Section 4.4 where their contribution to certain elasto-static problems is compared with other lattice types.

\subsection{Case 2: deformable nodes}

Analytical expressions for the mechanical behavior of the chiral lattice with deformable rings are difficult to define. Legitimate modeling of the rings requires either an approximate representation based on many straight members or nonlinear coupled equations for curved beams. In both cases, analytical solutions, if available at all, become unintelligible. A finite-element (FE) model of a unit cell like the one depicted in Fig. 1a is employed instead. Six independent strain states $\boldsymbol{\varepsilon}=\left\{\begin{array}{lllllll}\varepsilon_{11} & \varepsilon_{22} & \varepsilon_{12} & \varepsilon_{21} & \kappa_{13} & \kappa_{23}\end{array}\right\}^{T}$ are imposed numerically. The analyzed unit cell is depicted in Fig. 5, where the degrees of freedom at the boundaries are shown. The displacements at each boundary site are prescribed according to Eqs. (19)-(21), each associated with a distinct strain state. The applied values are listed in Table 1 for each considered case. Given the rotational symmetry of the lattice, the displacements of only three sites are reported. As for the case of rigid rings, rotations $\alpha_{i}(i=1,2, \ldots, 6)$ of the ligaments are associated with global deformations (Fig. 3$)$ and thus are to be distinguished from micro-rotations $\phi_{i}$. In Table 1 , these initially unknown macro-rotations are indicated as $\hat{\alpha}_{i}$. They are obtained by employing the same equation partitioning reported in Eq. (22), albeit in this case $\hat{\boldsymbol{K}}$ corresponds to the global stiffness matrix without rigid-ring kinematic constraints. In each case, the strain component is set to unity to evaluate the associated elastic constants. The elastic cross term $D_{12}$ is evaluated by setting $\varepsilon_{11}=\varepsilon_{22}=1$ in calculating $\Psi$ and subtracting the strain energy associated with $\varepsilon_{11}$ and $\varepsilon_{22}$. A similar procedure is used to evaluate $D_{34}$.

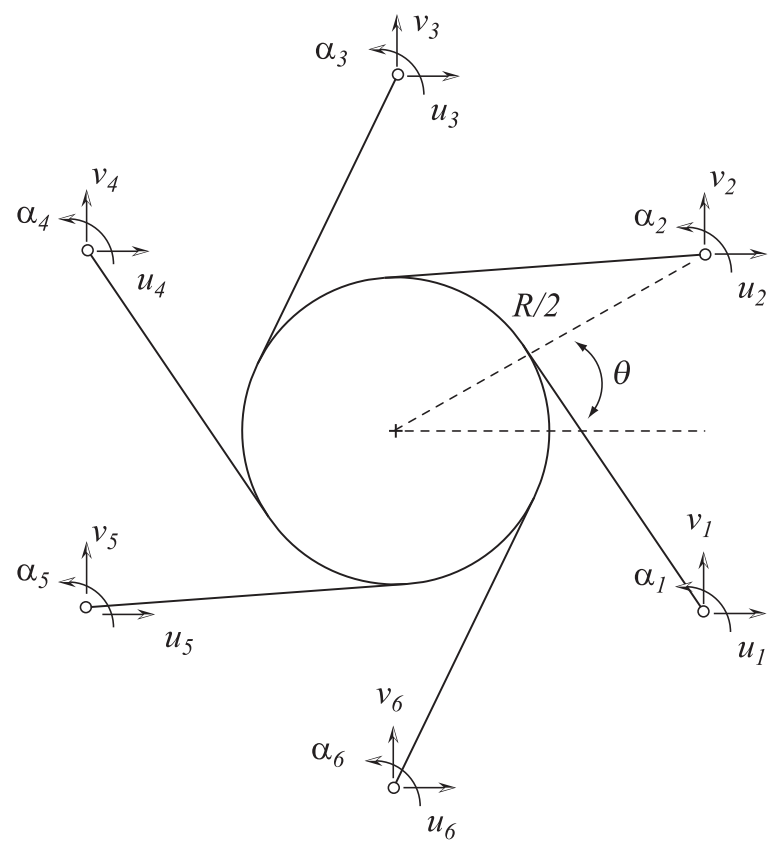

Fig. 5. FE model of the unit cell employed to study configurations with deformable rings with boundary degrees of freedom, prescribed for each considered strain state.

Table 1

Imposed displacements corresponding to six independent strain states.

\begin{tabular}{lllllll}
\hline & $\varepsilon_{11}$ & $\varepsilon_{22}$ & $\varepsilon_{12}$ & $\varepsilon_{21}$ & $\kappa_{13}$ & $\kappa_{23}$ \\
\hline$u_{1}$ & $R \cos \theta / 2$ & 0 & $-R \sin \theta / 2$ & 0 & 0 & 0 \\
$v_{1}$ & 0 & $-R \sin \theta / 2$ & 0 & $R \cos \theta / 2$ & 0 \\
$\alpha_{1}$ & $\hat{\alpha}_{1}$ & $\hat{\alpha}_{1}$ & $\hat{\alpha}_{1}+1 / 2$ & $\hat{\alpha}_{1}-1 / 2$ & 0 & $-R \cos \theta / 2$ \\
$u_{2}$ & $R \cos \theta / 2$ & 0 & $R \sin \theta / 2$ & 0 & 0 & 0 \\
$v_{2}$ & 0 & $R \sin \theta / 2$ & 0 & $R \cos \theta / 2$ & 0 \\
$\alpha_{2}$ & $\hat{\alpha}_{2}$ & $\hat{\alpha}_{2}$ & 0 & $\hat{\alpha}_{2}+1 / 2$ & 0 & 0 \\
$u_{3}$ & 0 & $R / 2$ & $R / 2$ & 0 & 0 \\
$v_{3}$ & 0 & $\hat{\alpha}_{3}$ & 0 & $\hat{\alpha}_{3}+1 / 2$ & $\hat{\alpha}_{3}-1 / 2$ & 0 \\
$\alpha_{3}$ & $\hat{\alpha}_{3}$ & & & 0 & 0 \\
\hline
\end{tabular}




\subsection{Comparison of rigid and deformable-ring configurations}

In order to provide a perception for the mechanical behavior of the chiral lattice, two particularly insightful elastic constants are depicted in Fig. 6 for two values of ligament aspect-ratio $(t / L)$, namely $t / L=[1 / 100,1 / 20]$. In all cases, the distance between rings $R=1 \mathrm{~m}$ and the ligament length $L$ varies according to $L / R$. Elastic constant $D_{11}$ from case 1 indicates a much stiffer mechanical behavior than for case 2 with respect to direct strains and for $L / R \neq 1$. This is not surprising as the strain energy related to ring deformations, a significant portion of the total for $L / R<0.9$, is neglected. $D_{11}$ associated with the rigid-ring model scales differently with aspect ratio compared to the values for case 2 . In the first case $D_{11} \propto(t / L)$, while in the second case $D_{11} \propto(t / L)^{3}$. The response to direct strains undergoes a dramatic change as rings become small. Above a critical value of topology parameter, dependent upon the ligament aspect ratio, the lattice's deformation mechanism changes (more dramatically with low ligament aspect ratio $(t / L)$ ) from being bending-dominated to one characterized by axial deflections of the ligaments. The rigid-ring configuration presents a much more abrupt (boundary-layer-like) transition between the two deformation mechanisms.

Elastic constant $D_{12}$ is crucial in defining auxetic behavior (Fig. 6b) as it determines direct-stress components perpendicular to a given direct strain component. The sign of Poisson's ratio $\left(v=D_{11} / D_{12}\right)$ is in fact determined by that of $D_{12}\left(D_{11}\right.$ must be positive for thermodynamic stability (Eringen, 2001)). In both case 1 and case $2, D_{12}$ decreases with increasing $L / R$ and a attains a minimum in terms of $L / R$ (strongest auxetic behavior) which is weakly dependent on $t / L$ that is the same nearly identical $L / R$ minima are observed for both considered values of $t / L$. The transition from bending to axial behavior (or auxetic to normal behavior) is determined by a change in sign in the numerator of Eq. (26). The topology parameter at which $v$ becomes positive can be estimated as $\tan ^{2} \beta\left[1-(t / L)^{2}\right]=\left[(t / L)^{2}-(t / L)^{4}\right]$. Stubby ligaments induce an earlier shift with respect to $L / R$. While this estimate is obtained from Eq. (26) for case 1 , it does provide a reasonable indication for such dramatic shift in behavior for case 2 also (Fig. 6b).

In terms of engineering constants (Fig. 7), the chiral lattice appears very compliant in agreement with the bendingdominated behavior. The value of Young's modulus defined for case 1 (Eq. (45)) is in significant error with respect to case 2 for $L / R<0.90$ (Fig. 7a), but it is an acceptable approximation as $L / R \rightarrow 1$ which leads to a significant increase in Young's modulus. The original model proposed by Prall and Lakes $(1997)\left(E / E s=\sqrt{(3)}(t / L)^{3}(L / r)^{2}\right)$ based on assumptions $1-5$ in Section 4 is also a reasonable approximation for topologies with very small rings.

In line with predictions in Prall and Lakes (1997), Spadoni (2008), Poisson's ratio (Fig. 7b) is approximately - 1 for case $1(\min [v]=-0.9984)$ with small $(t / L)$ and approaches $1 / 3$ as $L / R \rightarrow 1$. Of note, Poisson's ratio is nearly unchanged for $L / R \leq 0.90$, although it presents a weak dependance on $t / L$ (as for Eq. (46)). Deformable rings, however, yield a different behavior, where $v$ rapidly decreases to a minimum $(\min [v]=-0.9426$ for $t / L=1 / 100$ and $\min [v]=-0.8204$ for $t / L=1 / 20$ ) with respect to $L / R$ before increasing towards the positive value for the triangular lattice in a boundary-layer-like manner, especially for small values of $t / L$. A hexagonal packing of rings $(L / R \rightarrow 0$, Fig. $7 \mathrm{~b})$ is instead characterized by $v \approx 0.8$. For this configuration, obviously, the predictions of case 1 should be neglected.

The shear modulus (Fig. 7c) is significantly overestimated by the model with rigid rings. The shear modulus obtained from case 2 in fact does not scale with $t / L$ for the entire range of considered $L / R$ values but rather with $(t / L)^{3}$, suggesting that deformable rings yield a configuration whose shear stiffness is strongly affected by deformations of the rings

a

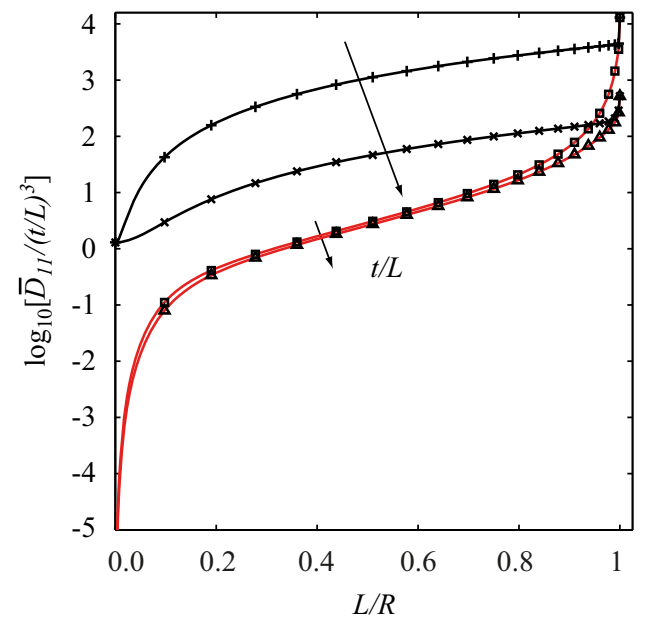

b

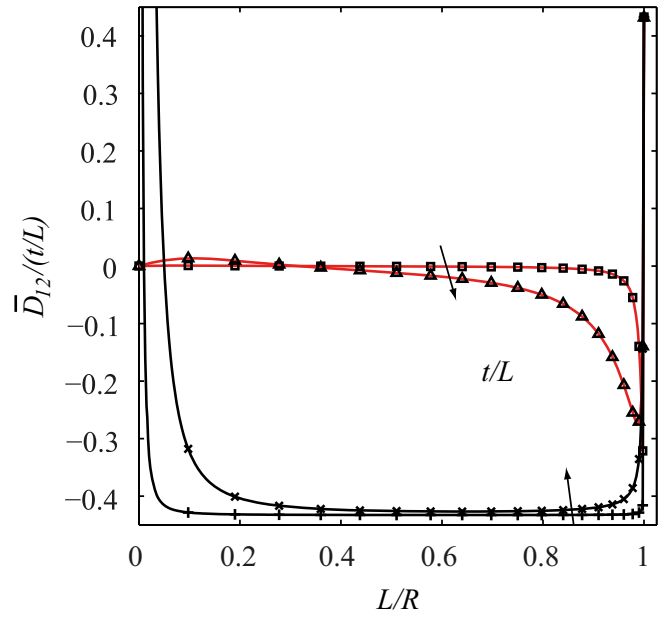

Fig. 6. Micropolar elastic constants $D_{11}$ (a) and $D_{12}$ (b) for a chiral lattice with two values of ligament aspect ratio $t / L=[1 / 100,1 / 20]$ with rigid (black lines,,$+ \times$ symbols) and deformable (red lines, $\square, \triangle$ symbols) rings. (For interpretation of the references to color in this figure legend, the reader is referred to the web version of this article.) 
a

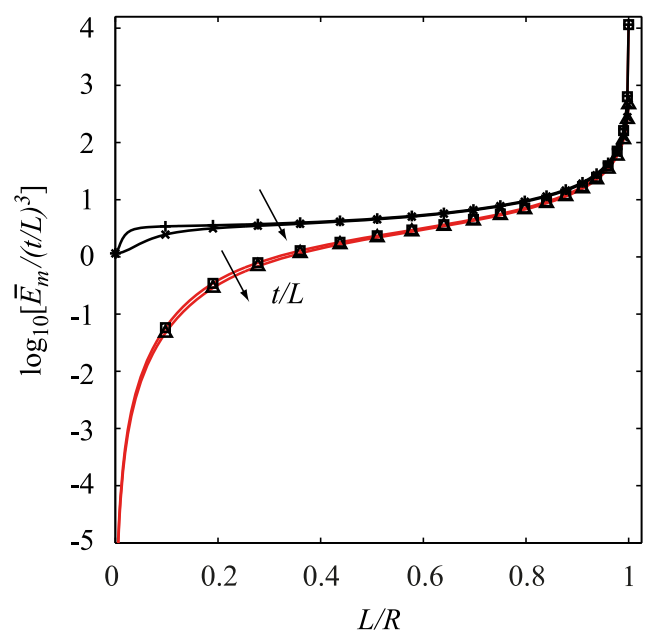

b

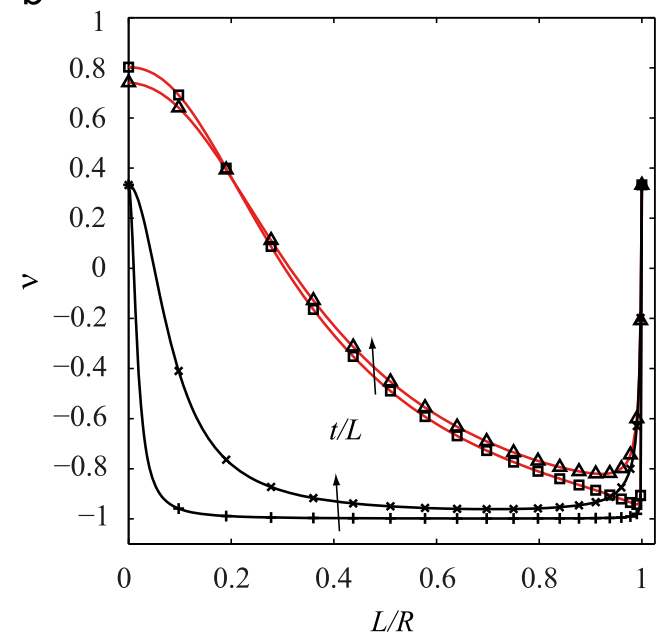

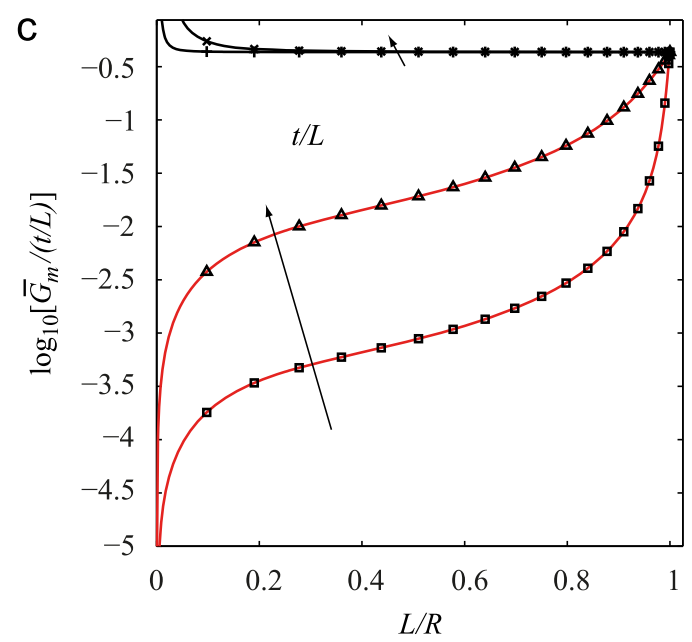

Fig. 7. Micropolar engineering constants for the chiral lattice with two values of ligament aspect ratio $t / L=[1 / 100,1 / 20]$ with rigid (black lines,,$+ \times$ symbols) and deformable (red lines, $\square, \triangle$ symbols) rings. Normalized Young's modulus (a), Poisson's ratio (b), and Shear modulus (c). (For interpretation of the references to color in this figure legend, the reader is referred to the web version of this article.)

themselves. As $L / R \rightarrow 1$, the shear modulus predicted by case 2 correctly approaches that of the triangular lattice from Eq. (48). A hexagonal packing of rings $(L / R \rightarrow 0$, Fig. $7 \mathrm{c})$ is found instead to be very compliant in shear.

The chiral lattice is a unique structural network from many points of view. This is also apparent comparing the derived constitutive law Eq. (24) with that for a general micropolar continuum. A 2D, isotropic, micropolar medium in plane stress has the constitutive law (Nakamura and Lakes, 1995)

$$
\left\{\begin{array}{l}
\sigma_{11} \\
\sigma_{22} \\
\sigma_{12} \\
\sigma_{21} \\
m_{13} \\
m_{23}
\end{array}\right\}=\left[\begin{array}{cccccc}
\frac{\left(2 \mu^{*}+\kappa\right)\left(2 \lambda+2 \mu^{*}+\kappa\right)}{\lambda+2 \mu^{*}+\kappa} & \frac{\lambda\left(2 \mu^{*}+\kappa\right)}{\lambda+2 \mu^{*}+\kappa} & 0 & 0 & 0 & 0 \\
\frac{\lambda\left(2 \mu^{*}+\kappa\right)}{\lambda+2 \mu^{*}+\kappa} & \frac{\left(2 \mu^{*}+\kappa\right)\left(2 \lambda+2 \mu^{*}+\kappa\right)}{\lambda+2 \mu^{*}+\kappa} & 0 & 0 & 0 & 0 \\
0 & 0 & \mu^{*}+\kappa & \mu^{*} & 0 & 0 \\
0 & 0 & \mu^{*} & \mu^{*}+\kappa & 0 & 0 \\
0 & 0 & 0 & 0 & \gamma & 0 \\
0 & 0 & 0 & 0 & 0 & \gamma
\end{array}\right]\left\{\begin{array}{l}
\varepsilon_{11} \\
\varepsilon_{22} \\
\varepsilon_{12} \\
\varepsilon_{21} \\
\kappa_{13} \\
\kappa_{23}
\end{array}\right\},
$$

where $\lambda$ and $\mu$ are the first and second Lamé constants $\left(\mu^{*}=\mu-\kappa / 2\right)$, while $\kappa$ and $\gamma$ are micropolar constants. From the analysis in Section 4.1, it can be verified that $\lambda=D_{12}\left(D_{34}+D_{33}\right) /\left(D_{34}+D_{33}-D_{12}\right)<0$. This characteristic is not found in the literature, and it is commonly assumed that $\lambda \geq 0$ (Eringen, 2001). Since $3 \lambda+2 \mu^{*}+\kappa=3 \lambda+D_{33}+D_{34}>0$, a necessary condition for thermodynamic stability (Eringen, 2001; Lakes and Benedict, 1982), an auxetic micropolar continuum in plane stress can have a negative first Lamé constant. The configuration with deformable rings also has $\lambda<0$ and 
$3 \lambda+2 \mu^{*}+k \geq 0$. This is consistent with the fact that both configurations have $\bar{G}_{m}>\bar{E}_{m}$. As a note, Eringen's bounds for stability mentioned here are expressed in terms of $\mu^{*}$ instead of $\mu$ in accordance with the observations by Cowin (1970).

\subsection{Comparison of chiral and traditional lattices}

It is helpful to compare the mechanical behavior of the chiral lattice to that of other periodic cellular solids both in terms of engineering and micropolar constants. Square, triangular and hexagonal lattices are common topologies encountered in physical models and engineering applications and their static behavior is discussed in Dempsey et al. (2005), Gibson and Ashby (1997), Wang et al. (2005), Wang and McDowell (2004), among others. The equivalent mechanical properties for such topologies in terms of geometric parameters are listed in Table 2. The results reported here, as for previous sections, have been obtained for a wall thickness $t=0.01 \mathrm{~m}$ and distance between ring centers $R=1 \mathrm{~m}$. The ligament length $L$ varies with $L / R$. The resulting relative density $\bar{\rho}$ from Eq. (4) for a topology parameter $0.0001 \leq L / R \leq 0.9999$ is shown in Fig. 8a. With the relative density at hand, it is possible to compare the mechanical properties of the chiral lattice from case 2 with those of square, triangular and hexagonal lattices. Young's modulus for each configuration is presented in Fig. 8b. The chiral lattice features the lowest Young's modulus for $0.0001 \leq L / R \leq 0.98$, while the square lattice is the least compliant. This is reasonable as the square lattice is dominated by axial deformations. The square lattice, on the other hand, features the lowest shear modulus, while the triangular lattice is the stiffest in shear. The chiral lattice performs better in shear than square and hexagonal lattices for $L / R>0.83$ and its shear modulus approaches that of the triangular topology as $L / R \rightarrow 1$, in agreement with the earlier findings of Eq. (48). It is remarkable that the shear modulus of the chiral lattice is significantly higher than both square and hexagonal lattices for $L / R \leq 0.98$ where all three topologies have a bending-dominated deformation mechanism.

Two additional constants, the characteristic length $l_{c}$ and the coupling number $N$ (from Eqs. (24) and (51) and Nakamura and Lakes, 1995),

$$
\begin{aligned}
& l_{c}^{2}=\frac{\gamma}{2\left(2 \mu^{*}+\kappa\right)}=\frac{\gamma}{4 G_{m}}, \\
& N^{2}=\frac{\kappa}{2\left(\mu^{*}+\kappa\right)}
\end{aligned}
$$

indicate the nature and significance of non-classical phenomena in the response of a medium with microstructure, here recast in terms of Lamé constants and micropolar parameters $\gamma$ and $\kappa$. The characteristic length determines the microrotation stiffness (Eq. (52)) and it introduces size effects in the response of a medium. More about this is discussed below where different topologies are compared. The coupling number $0 \leq N \leq 1$ indicates the mechanism producing micro-

Table 2

Mechanical properties of common lattice topologies (Wang and McDowell, 2004).

\begin{tabular}{lllll}
\hline Topology & $\bar{\rho}$ & $E / E_{s}$ & $G / E_{s}$ & $v$ \\
\hline Square & $2 t$ & $\frac{1}{2} \bar{\rho}$ & $\frac{1}{16} \bar{\rho}^{3}$ & $\frac{1}{2} v_{s} \bar{\rho}$ \\
Triangular & $2 \sqrt{3} \frac{t}{L}$ & $\frac{1}{3} \bar{\rho}$ & $\frac{1}{8} \bar{\rho}$ & $1 / 3$ \\
Hexagonal & $\frac{2}{\sqrt{3}} \frac{t}{L}$ & $\frac{3}{2} \bar{\rho}^{3}$ & $\frac{3}{8} \bar{\rho}^{3}$ & 1.0 \\
\hline
\end{tabular}

a

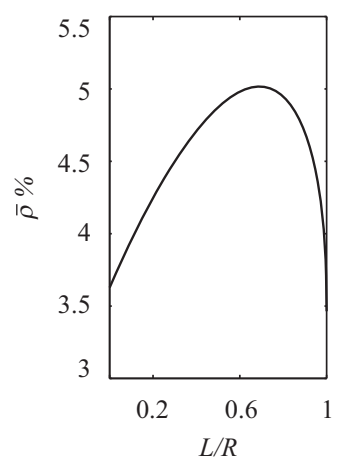

b

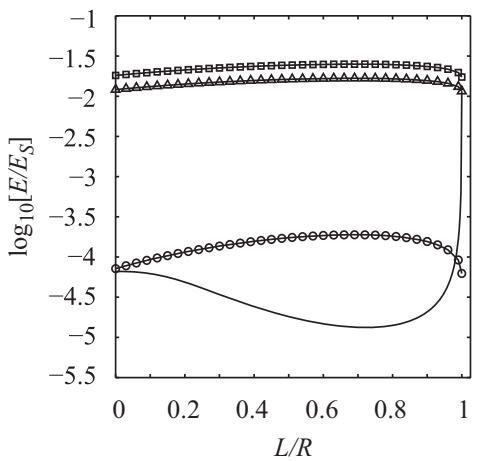

C

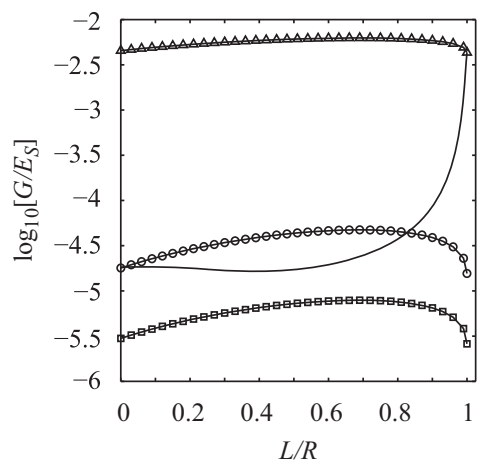

Fig. 8. Relative density $\bar{\rho}$ (a) and comparison of Young's modulus (b) and shear modulus (c) for various periodic topologies: chiral lattice (-), square lattice $(-\square-)$, triangular lattice $(-\triangle-)$ and hexagonal lattice $(-\circ-)$. 
rotations and the contribution of the skew-symmetric part of the stress tensor or non-classical effects (Cowin, 1970; Eringen, 2001). The two extreme cases $N=0$ and $N=1$ denote classical-elasticity behavior and the coinciding of microrotations and macro-rotations respectively (Cowin, 1970). The latter is known as couple-stress (Cowin, 1970) or Mindlin theory (Eringen, 2001; Mindlin, 1963), and it is a special case of micropolar elasticity with constrained micro-rotations or $\gamma \rightarrow \infty$ (Cowin, 1970). $0<N<1$ instead denotes micropolar behavior (Cowin, 1970).

The characteristic length and coupling number for the micropolar behavior described by the constitutive model of Eqs. (25)-(34) and Eq. (49) (rigid rings) and that computed from Section 4.2 (deformable rings) are shown in Fig. 9. As a note, the values predicted for the rigid-ring configuration are meaningless in the limit $L / R \rightarrow 0$ which represents a perfectly rigid lattice. The coupling number $N$ is large for both rigid and deformable-ring configurations, especially for low values of $L / R$. In the limiting case $L / R=0$, our analysis indicates that micro and micro-rotations coincide for a hexagonal packing of deformable rings ( $N=1$ in Fig. 9b). The behavior of this lattice is then described by couple-stress or Mindlin theory (Mindlin, 1963). So far couple-stress-elasticity behavior has been assumed a priori for certain configurations (Banks and Sokolowski, 1968) (incorrectly in this case, see remarks in Bažant and Christensen, 1972), or it was concluded based on experimental measurements (Yang and Lakes, 1981, 1982). A hexagonal packing of rings therefore may provide a useful test bed to experimentally measure the implications of Mindlin theory. The coupling number for a triangular lattice is instead very small suggesting classical-elasticity behavior. For rigid rings in fact $N \rightarrow 2 t^{2} / L^{2}$ as $L / R \rightarrow 1$ from Eq. (50), a trend that is indicative of deformable-ring configurations also. This is reasonable as a triangular lattice is dominated by axial deformations. Micropolar effects, i.e. micro-rotations, are thus a higher-order effect. The coupling number also determines the solution of an elastic half-space loaded by a concentrated load (Eringen, 2001; Khan and Dhaliwal, 1977) (in their notation $k^{2}=4 N^{2}$ ).

A number of analytical (Cowin, 1970; Diebels and Steeb, 2002; Eringen, 2001; Gauthier and Jahsman, 1975; Mindlin, 1963; Onck et al., 2001) numerical (Chen and Fleck, 2002; Diebels and Steeb, 2002; Onck et al., 2001) and experimental studies of foams (Andrews et al., 2001; Chen and Fleck, 2002; Lakes, 1983) and human bone (Yang and Lakes, 1981, 1982) have indicated various size effects in the compliance of media with microstructure, in addition to stress concentration in the vicinity of boundaries and discontinuities. With the exception of Onck et al. (2001), no connection between microstructure type and specific behavior is provided and the general contribution of the lattice parameter (or average cell size for stochastic configurations) and length scale $l_{c}$ are discussed. In comparing previous experimental work and theoretical models with the behavior of the chiral lattice, it is indicative to consider the micropolar behavior of classic configurations summarized in Table 3. Deformation mechanisms associated with shearing and denoted by bending of internal members result in high coupling numbers (important contribution of micro-rotations) and $l_{c}$ scales with the internal members' length (which may or may not be the lattice parameter). This is evident for square and hexagonal configurations. The high values of $l_{c}$ derived here (Eq. (49) and Fig. 9) confirm that bending deformations of internal members dominate the shearing response of the chiral lattice. The triangular lattice responds to shear with axial deformations and thus $l_{c}$ and $N$ are small and are proportional to the internal members' wall thickness $t$ (see Tables 2 and 3).

Yang and Lakes (1982) find that micropolar effects (significant stiffening) can be measured for specimen with a diameter 10 times the characteristic length or smaller, and for experiments on human bone, Lakes (1983) and Yang and Lakes (1982) find that the characteristic length $l_{c}$ is comparable "to the size of the dominant structural elements". In view

a

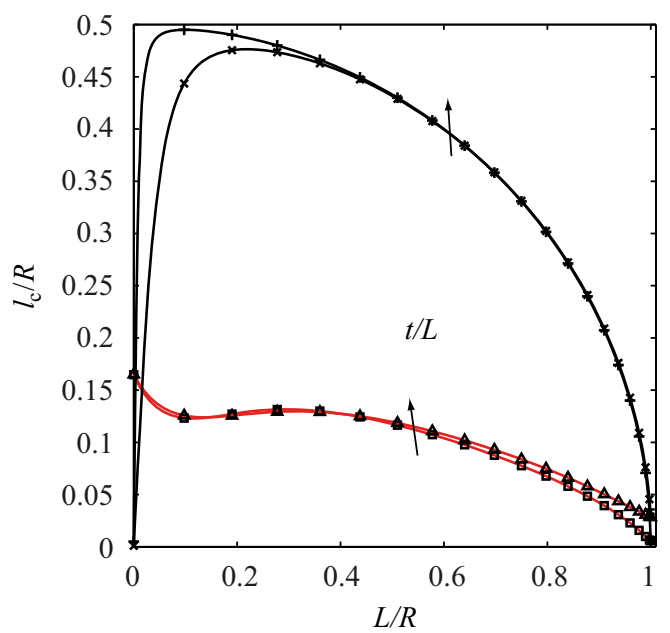

b

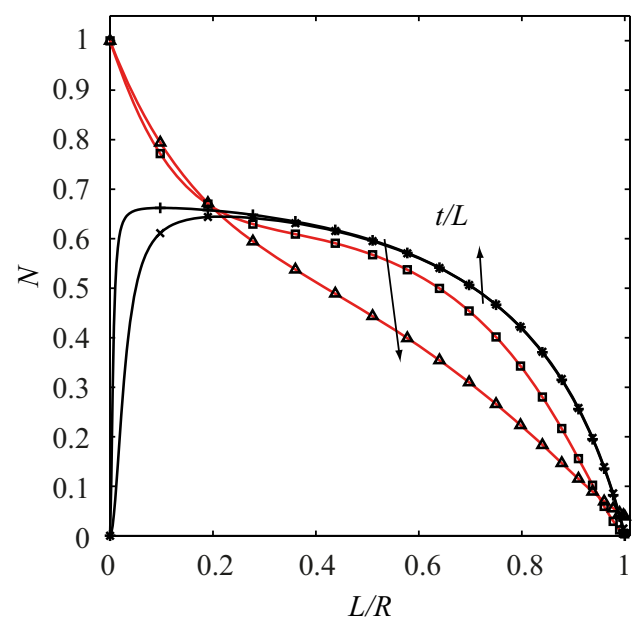

Fig. 9. Characteristic length (a) and coupling number (b) of the chiral lattice with two values of ligament aspect ratio $t / L=[1 / 100,1 / 20]$, with rigid (black lines,,$+ \times$ symbols) and deformable (red lines, $\square, \triangle$ symbols) rings. (For interpretation of the references to color in this figure legend, the reader is referred to the web version of this article.) 
Table 3

Micropolar length scale and coupling number of common lattice topologies (Banks and Sokolowski, 1968; Dos Reis and Ganghoffer, 2011; Wang and McDowell, 2004).

\begin{tabular}{lll}
\hline Topology & $l_{c}^{2}$ & $N^{2}$ \\
\hline Square & $\frac{L^{2}}{24}$ & $\frac{1}{2}$ \\
Triangular & $\frac{t^{2}}{3\left(1-t^{2} / L^{2}\right)}$ & $\frac{2 t^{2} / L^{2}}{\left(1+3 t^{2} / L^{2}\right)}$ \\
Hexagonal & $\frac{L^{2}}{48}\left(1+t^{2} / L^{2}\right)$ & $\frac{\left(1+t^{2} / L^{2}\right)}{\left(3+t^{2} / L^{2}\right)}$ \\
\hline
\end{tabular}

a

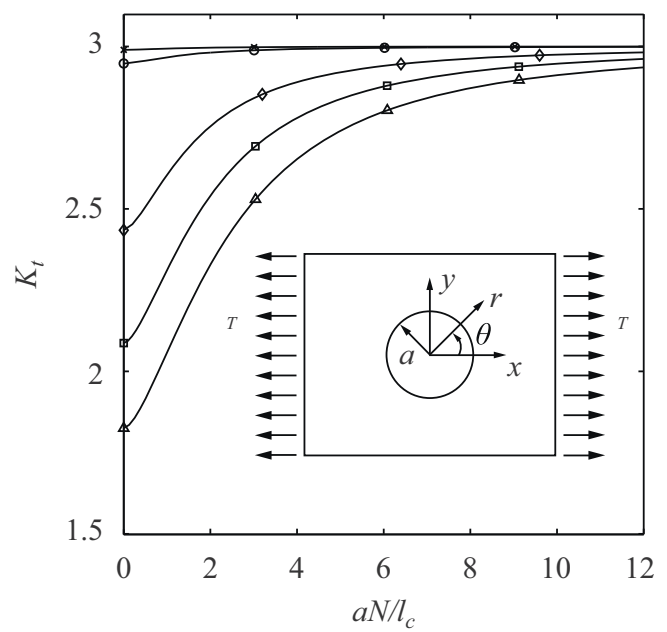

b

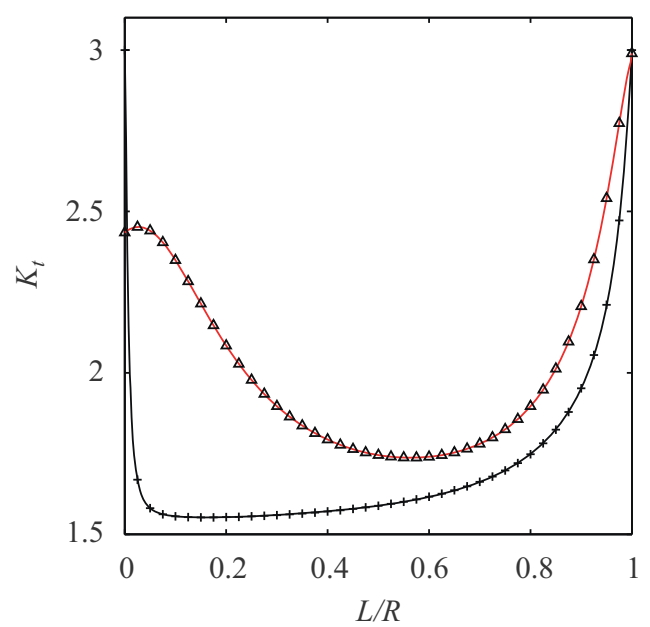

Fig. 10. Stress concentration $K_{t}$ as a function of cylindrical-cavity radius $a(L / R=0-\diamond-, 0.75-\triangle-, 0.87-\square-, 0.995-\circ-, 1-\times-)$ (a) and as a function of $L / R\left(a N / l_{c}=0\right)(\mathrm{b})$, given the micropolar properties of the chiral lattice with deformable rings (a), and deformable $(-\Delta-)$ and rigid rings $(-+-)$ (b) with $t / L=1 / 100$ and $R=1 \mathrm{~m}$.

of the analysis above, this may shed some light on the microstructural characteristics of human bone. Numerical models and experimental measurements by Chen and Fleck (2002) also find that size effects are directly proportional to the cell edge length. The numerical models by Chen and Fleck (2002) considered hexagonal cells and stochastic configurations as perturbations of hexagonal cells. Numerical investigations by Diebels and Steeb (2002) find that boundary-layers and size effects observed in the deformation and stress distribution for a cellular solid loaded in shear arise because of "obstructed" rotations at the boundaries, mimicking clamped boundary conditions. Given the strong micropolar behavior of the chiral lattice, significant stiffening and boundary layers are expected in analogy with numerical models and experiments above.

The length $l_{c}$ is weakly dependent on $t / L$ for both rigid and deformable-ring configurations, and it is significantly higher for large rigid rings, in which case it is approximately half the lattice parameter $R$ (Fig. $9 \mathrm{a}$ ). As $L / R$ increases, $l_{c}$ becomes very small, several orders of magnitude smaller than $R$ ( $\approx 1 / 3 t^{2}$ from Eq. (49), in agreement with Table 3 ). Deformable rings yield lower values of $l_{c}$. This is reasonable as $l_{c}$ affects the decay rate of internal stresses from the values of tractions at the boundaries, and for case 2, rings do contribute in storing strain energy induced by boundary loading. Adopting the conclusions by Chen and Fleck (2002), Diebels and Steeb (2002), Lakes (1983), and Yang and Lakes (1982), chiral lattices with small (or deformable) rings are expected to produce less significant size effects than their counterparts with large (or deformable) rings.

A classic problem that elucidates boundary effects for micropolar media as functions of $l_{c}$ and $N$ is the stress concentration around a cylindrical cavity of radius $a$ in a field of uniaxial stress $T$ (Cowin, 1970; Eringen, 2001; Mindlin, 1963). The maximum tangential stress $\sigma_{\theta \theta}(r, \theta)$ is found as (Eringen, 2001)

$$
\begin{aligned}
& \sigma_{\theta \theta}(r, 0)=T \frac{\left(3+F_{1}\right)}{\left(1+F_{1}\right)}, \\
& F_{1}=8(1-v) N^{2}\left[4+\frac{a^{2} N^{2}}{l_{c}^{2}}+\frac{2 a N}{l_{c}} \frac{K_{0}\left(a N / l_{c}\right)}{K_{1}\left(a N / l_{c}\right)}\right]^{-1},
\end{aligned}
$$

where $K_{0}$ and $K_{1}$ are modified Bessel functions of the second kind. The concentration factor $K_{t}=\sigma_{\theta \theta}(r, 0) / T$ is shown in Fig. 10. Configurations with large rings have a high coupling number $(N \rightarrow 1$ as $L / R \rightarrow 0$ in Fig. $9 \mathrm{~b})$. From the analysis in 
Cowin (1970), Eringen (2001), and Mindlin (1963) however this should coincide with the lowest stress-concentration factor, which instead takes place here for $L / R=\approx 0.57$ and $N=0.74$. In this case, however, Poisson's ratio also plays a role (Eq. (55)), and it varies with $L / R$. As $L / R \rightarrow 1$, the stress concentration for the classical-elasticity solution is recovered.

\section{Summary}

The objective of this work is the definition of an equivalent, micropolar, continuum model to improve the description of the elasto-static behavior of a chiral auxetic lattice. This is a structural network composed of rings connected by tangent ligaments. Previous analyses indicated that the chiral lattice is transversely isotropic with an in-plane Poisson's ratio exactly equal to -1 , yielding an undefined shear modulus and constitutive law in general. This inhibits attempts to include this topology in optimization studies for structural applications.

This article introduces a micropolar continuum model that is derived from a complex unit cell which, unlike previous work, includes all topologically distinct components. The analysis is based on two cases, one with rigid rings, and a second case where a numerical model is employed to analyze the contribution of deformable rings. The derived continuum model is a continuous function of the topology parameter $L / R$ which allows investigating the mechanical behavior of significantly different configurations, ranging from a hexagonal packing of deformable rings to the triangular lattice. Auxetic behavior is confirmed for both rigid and deformable-ring configurations, except for the limiting case $L / R \rightarrow 1$. The latter corresponds to the triangular lattice whose behavior is dominated by axial deformation of its internal components. For $L / R \neq 1$ instead, the chiral lattice is dominated by bending deformation of the ligaments. The ideal, rigid-ring case indicates that mechanical properties vary with $L / R$ but undergo a boundary-layer like behavior as $L / R \rightarrow 1$. Poisson's ratio is found to depend on ligament aspect ratio $t / L$ and can be -1 only for $t / L=0$. The indeterminacy in the constitutive law of an equivalent continuum is thus removed.

Our analysis also indicates that the shear modulus of the auxetic chiral lattice with rigid rings is equal to that of the triangular lattice. This is an outstanding characteristic for a bending-dominated lattice, but it indicates that the deformation mechanism necessary to achieve strong isotropic, auxetic behavior limits the shear stiffness to that of a medium with axially dominated deformations. This in turn restricts auxetic materials, at least in two dimensions, to be very efficient systems to resist shear relatively to bending-dominated configurations and not in an absolute sense. The shear modulus is found to be much lower if the contribution of deformable rings is included. In this case, Poisson's ratio is negative for most values of $L / R$ but attains a minimum, which depends on ligament aspect ratio, before increasing towards the value of the triangular lattice $(v=0.33)$. The shear modulus corresponding to the minimum Poisson's ratio is naturally higher than Young's modulus and it is higher than that for other common lattices like the square and hexagonal configurations. The shear modulus correctly approaches its upper limit corresponding to the triangular lattice. Except for $L / R \rightarrow 1$, Young's modulus is the lowest of all common lattices.

The micropolar response of the chiral lattice is also unique in that it has the highest characteristic length scale $l_{c}$ of all known lattice topologies. Our models also indicate that hexagonal distributions of deformable rings have a coupling number $N=1$. This is the first lattice reported in the literature for which couple-stress or Mindlin theory is necessary rather than being adopted a priori. Finally, it is found that the chiral lattice, both with rigid and deformable rings, has a negative first Lamé constant without violating bounds required for thermodynamic stability. This is an outstanding characteristic that has never been reported before.

\section{References}

Alderson, K., Pickles, A., Neale, P., Evans, K., 1994. Auxetic polyethylene: the effect of a negative Poisson's ratio on hardness. Acta Metall. Mater. 42 (7), 2261-2266

Andrews, E.W., Gioux, G., Onck, P., Gibson, L.J., 2001. Size effects in ductile cellular solids. Part II: experimental results. Int. J. Mech. Sci. 43 (3), 701-713. Banks, C.B., Sokolowski, M., 1968. On certain two-dimensional applications of the couple stress theory. Int. J. Solids Struct. 4 (1), $15-29$.

Bažant, Z.P., Christensen, M., 1972. Analogy between micropolar continuum and grid frameworks under initial stress. Int. J. Solids Struct. 8 (3), 327-346. Chen, C., Fleck, N.A., 2002. Size effects in the constrained deformation of metallic foams. J. Mech. Phys. Solids 50 (5), $955-977$.

Choi, J.B., Lakes, R.S., 1995. Analysis of elastic modulus of conventional foams and of re-entrant foam materials with a negative Poisson's ratio. Int. J. Mech. Sci. 37 (1), 51-59.

Cowin, S.C., 1970. An incorrect inequality in micropolar elasticity theory. Z. Angew. Math. Phys. 21 (3), $494-497$.

Dempsey, B.M., Eisele, S., McDowell, D.L., 2005. Heat sink applications of extruded metal honeycombs. Int. J. Heat Mass Transfer 48, 527-535.

Deshpande, V.S., Ashby, M.F., Fleck, N.A., 2001. Foam topology bending versus stretching dominated architectures. Acta Mater. 49, 1035-1040.

Diebels, S., Steeb, H., 2002. The size effect in foams and its theoretical and numerical investigation. Proc. Roy. Soc. A-Math. Phys. 458 (2028), 2869.

Dos Reis, F., Ganghoffer, J.F., 2011. Construction of Micropolar Continua from the Homogenization of Repetitive Planar Lattices. Springer, pp. 193-217 (Chapter 9).

Doyoyo, M., Hu, J.W., 2006. Plastic failure analysis of an auxetic foam or inverted strut lattice under longitudinal and shear loads. J. Mech. Phys. Solids 54 (7), 1479-1492.

Eringen, A.C., 2001. Microcontinuum Field Theories: I Foundations and Solids. Springer, New York.

Evans, K., 1990. Tailoring the negative Poisson ratio. Chem. Ind.-London 20, 654-657.

Evans, K.E., Alderson, A., 2000. Auxetic materials: functional materials and structures from lateral thinking!. Adv. Mater. 12 (9), $617-628$.

Gaspar, N., Ren, X.J., Smith, C.W., Grima, J.N., Evans, K.E., 2005. Novel honeycombs with auxetic behaviour. Acta Mater. 53 (8), $2439-2445$

Gauthier, R.D., Jahsman, W.E., 1975. A quest for micropolar elastic constants. J. Appl. Mech. 42, 369.

Gibson, L.J., Ashby, M.F., 1997. Cellular Solids: Structure and Properties, 2nd ed. Cambridge University Press.

Grima, J.N., Gatt, R., Farrugia, P.S., 2008. On the properties of auxetic meta-tetrachiral structures. Phys. Status Solidi B 245, 511-520.

Khan, S.M., Dhaliwal, R.S., 1977. Axisymmetric problem for a half-space in the micropolar theory of elasticity. J. Elasticity 7 (1), $13-32$. 
Kumar, R.S., McDowell, D.L., 2004. Generalized continuum modeling of 2-D periodic cellular solids. Int. J. Solids Struct. 41 (26), 7399-7422.

Lakes, R.S., 1983. Size effects and micromechanics of a porous solid. J. Mater. Sci. 18 (9), 2572-2580.

Lakes, R.S., 1991. Deformation mechanisms in negative Poisson's ratio materials: structural aspects. J. Mater. Sci. 26, 2287-2292.

Lakes, R.S., 1987. Foam structures with a negative Poisson's ratio. Science 235, 1038-1040.

Lakes, R.S., Benedict, R.L., 1982. Noncentrosymmetry in micropolar elasticity. Int. J. Eng. Sci. 20 (10), 1161-1167.

Lakes, R.S., Elms, K., 1993. Indentability of conventional and negative Poisson's ratio foams. J. Compos. Mater. 27 (12), $1193-1202$.

Lakes, R.S., Witt, R., 2002. Making and characterizing negative Poisson's ratio materials. Int. J. Mech. Eng. Educ. 30 (1), 50-58.

Love, A.E.H., 1927. A Treatise on the Mathematical Theory of Elasticity, 4th ed. Dover Publications.

Mindlin, R.D., 1963. Influence of couple-stresses on stress concentrations. Exp. Mech. 3 (1), 1-7.

Nakamura, S., Lakes, R.S., 1995. Finite element analysis of saint-venant end effects in micropolar elastic solids. Eng. Comput. 12, 571-587.

Onck, P.R., Andrews, E.W., Gibson, L.J., 2001. Size effects in ductile cellular solids. Part I: modeling. Int. J. Mech. Sci. 43 (3), 681-699.

Prall, D., Lakes, R.S., 1997. Properties of a chiral honeycomb with a Poisson's ratio of -1. Int. J. Mech. Sci. 39 (3), 305-314.

Smith, C.W., Lehman, F., Wootton, R.J., Evans, K.E., 1999. Strain dependent densification during indentation in auxetic foams. Cell. Polym. 18 (2), $79-101$.

Smith, C.W., Grima, J.N., Evans, K.E., 2000. A novel mechanism for generating auxetic behaviour in reticulated foams: missing rib foam model. Acta Mater. 48 (17), 4349-4356.

Spadoni, A., August 2008. Application of Chiral Cellular Materials for the Design of Innovative Components. Ph.D. Thesis, Georgia Institute of Technology. Spadoni, A., Ruzzene, M., Gonella, S., Scarpa, F., 2009. Phononic properties of hexagonal chiral lattices. Wave Motion 46 (7), 435-450.

Tanaka, H., Shibutani, Y., 2009. In-plane mechanical behaviors of $2 \mathrm{~d}$ repetitive frameworks with four-coordinate flexible joints and elbowed beam members. J. Mech. Phys. Solids 57 (9), 1485-1499.

Theocaris, P.S., Stavroulakis, G.E., Panagiotopoulos, P.D., 1997. Negative Poisson's ratios in composites with star-shaped inclusions: a numerical homogenization approach. Arch. Appl. Mech. 67, 274-286.

Wang, A.J., McDowell, D.L., 2004. In-plane stiffness and yield strength of periodic metal honeycombs. J. Eng. Mater.-Trans. ASME 126, 137-156.

Wang, A.J., Kumar, R.S., McDowell, D.L., 2005. Mechanical behavior of extruded prismatic cellular metals. Mech. Adv. Mater. Struc. 12, 185-200.

Yang, D.U., Huang, F.Y., 2001. Analysis of Poisson's ratio for a micropolar elastic rectangular plate using the finite element method. Eng. Comput. 18 (7), $1012-1030$

Yang, J.F.C., Lakes, R.S., 1981. Transient study of couple stress effects in compact bone: torsion. J. Biomech. Eng.-Trans. ASME $103,275$.

Yang, J.F.C., Lakes, R.S., 1982. Experimental study of micropolar and couple stress elasticity in compact bone in bending. J. Biomech. 15 (2), 91-98.

Yang, W., Li, Z.M., Shi, W., Xie, B.H., Yang, M.B., 2004. Review on auxetic materials. J. Mater. Sci. 39 (10), 3269-3279. 